\title{
25.
}

\section{NOTE ON THE THEOREM CONTAINED IN PROFESSOR LIPSCHITZ'S PAPER.}

\author{
[American Journal of Mathematics, I. (1878), pp. 341-343.]
}

I THINK it may be useful to state the principle to which the theorem demonstrated in the preceding paper leads, in the shape in which it has always presented itself to my mind, but which I found difficult to express when writing under the constraint of a foreign language.

It amounts simply to the statement that in a prepared form just as the variables $(x, y, z, \ldots)$ are contragredient to their symbolic inverses $\left(\frac{d}{d x}, \frac{d}{d y}, \frac{d}{d z}, \ldots\right)$ so the coefficients $(a, b, c, \ldots)$ are contragredient to theirs $\left(\frac{d}{d a}, \frac{d}{d b}, \frac{d}{d c}, \ldots\right)$; the latter statement in fact includes the former inasmuch as the so-called variables may be regarded as the coefficients of an auxiliary linear form.

In applying this principle it is expedient to enlarge our conception of invariants, covariants, etc., and to predicate invariance of functions not only of quantities ordinarily so termed, but of their symbolic inverses, or of functions in which quantities and operators enter conjointly*. To draw the

* It was through this idea that I was originally led to an intuitive perception of the theorems concerning the prepared form. For suppose $\left(a, b, c, \ldots l \gamma(x, y, z)^{n}\right.$ to be any prepared form; then if $x^{\prime}, y^{\prime}, z^{\prime} ; x^{\prime \prime}, y^{\prime \prime}, z^{\prime \prime}$ are cogredient with $x, y, z$, and we operate upon the given form with

$$
\left(\dot{a}, \dot{b}, \dot{c}, \ldots i \chi_{\chi} y^{\prime} z^{\prime \prime}-y^{\prime \prime} z^{\prime}, z^{\prime} x^{\prime \prime}-z^{\prime \prime} x^{\prime}, x^{\prime} y^{\prime \prime}-x^{\prime \prime} y^{\prime}\right)^{n},
$$

the result is the $n$th power of the determinant $\left|\begin{array}{lll}x & y & z \\ x^{\prime} & y^{\prime} & z^{\prime} \\ x^{\prime \prime} & y^{\prime \prime} & z^{\prime \prime}\end{array}\right|$ which is a covariant. Hence we may conclude that the operator is a covariant; just as, if a covariant multiplied by any form is a covariant, we may conclude that the multiplier must be so too. Consequently

$$
\left(\dot{a}, \dot{b}, \dot{c}, \ldots \dot{i} \chi_{\curlywedge} x, y, z\right)^{n}
$$

is a contravariant, and the same reasoning will apply whatever may be the number of variables. I originally used two forms, one the ordinary form for $(a, b, c, \ldots l\rangle x, y, z, \ldots)$ and the other the 
conclusions which flow from this conception, we have only to add the rule that all combinations of invariants, or of covariants, or of contravariants, etc., are themselves invariants, or covariants, or contravariants, etc., respectively. We may then state that the effect of substituting in a covariant or contravariant (to a prepared form) in place of the variables, or in place of the coefficients, the symbolic inverses of the one or the other, is to reverse their character and convert covariants into contravariants and vice versa, leaving of course the character of invariants unaltered; and I may remark incidentally that we are thus provided with a means of making any two invariants operate on each other so as to produce a third, a mode of operation which was not possible previous to the introduction of the prepared form*.

Moreover, the word combination must be taken in its widest sense, as there is more than one mode of combination possible. For example, if $F, G, H$ are covariantive and $\Phi$ a contravariantive function of $(a, b, c, \ldots ; x, y, z, \ldots)$, where $a, b, c, \ldots$ are the coefficients and $x, y, z, \ldots$ the variables of a prepared form, we have of course $\Phi\left(\frac{d}{d a}, \frac{d}{d b}, \ldots ; x, y, \ldots\right) F$ and

$$
\Phi\left(a, b, \ldots ; \frac{d}{d x}, \frac{d}{d y}, \ldots\right) F \text { and } G\left(\frac{d}{d a}, \frac{d}{d b}, \ldots ; \frac{d}{d x}, \frac{d}{d y}, \ldots\right) F
$$

all of them covariants. This may be termed an external mode of combination, but we shall equally have covariants derived by an internal mode of combination, for example

$$
\begin{gathered}
\Phi\left(\frac{d F}{d a}, \frac{d F}{d b}, \ldots ; x, y, \ldots\right), \quad \Phi\left(a, b, \ldots ; \frac{d F}{d x}, \frac{d F}{d y}, \ldots\right), \\
H\left(\frac{d F}{d a}, \frac{d F}{d b}, \ldots ; \frac{d G}{d x}, \frac{d G}{d y}, \ldots\right)
\end{gathered}
$$

will also be covariants.

ordinary form divested of its numerical coefficients for $\left(\dot{a}, \ldots \nmid y^{\prime} z^{\prime \prime}-y^{\prime \prime} z^{\prime}, \ldots\right)^{n}$, and of course with the same result.

The reasoning is perhaps not absolutely rigorous, but sufficiently so to bring conviction of the fact to be established. Of course when we have proved that $\left(\dot{a}, \dot{b}, \dot{c}, \ldots i \chi_{\curlyvee} x, y, z\right)^{n}$ is a contravariant, it follows more generally that if $(A, B, C, \ldots \succ x, y, z, \ldots)^{N}$ is a covariant

$$
(\dot{A}, \dot{B}, \dot{C}, \ldots \curlywedge \backslash x, y, z, \ldots)^{N},
$$

where $\dot{A}, \dot{B}, \dot{C}, \ldots$ are the same functions of $\dot{a}, \dot{b}, \dot{c}, \ldots$ as $A, B, C, \ldots$ are of $a, b, c, \ldots$, will be a contravariant and vice versa.

* The case may be stated thus: previous to the introduction of the prepared form, invariants of systems could be made to operate upon invariants solely through the instrumentality of the coefficients of the linear forms of the system; since its introduction the same operation may be made to take effect through the instrumentality of the coefficients of all the forms, linear or non-linear, indiscriminately. The first named mode of operation is equivalent to the hyperdeterminantive method, which includes that of Ueberschiebung; the latter transcends the sphere of hyperdeterminants. 
So again it may be observed that these modes of combination admit of being applied in more than one way: thus, to confine ourselves for a moment to the case of two forms, their external operation on each other may be simple, or concurrent, or reciprocal: simple when in one of them one set of quantities are converted into operators, concurrent when both sets are so converted, but reciprocal when in one of the two forms the variables and in the other the coefficients undergo such conversion. As an example, suppose we take the prepared form $a x^{3}+\ldots+d y^{3}$, and its skew covariant

$$
\left(a^{2} d+\ldots\right) x^{3}+\ldots-\left(a d^{2}+\ldots\right) y^{3}
$$

We may combine the contravariant

$$
\left(\frac{d}{d a} x^{3}+\ldots+\frac{d}{d d} y^{3}\right)^{2}
$$

with the contravariant

$$
\left(a^{2} d+\ldots\right)\left(\frac{d}{d x}\right)^{3}+\ldots-\left(a d^{2}+\ldots\right)\left(\frac{d}{d y}\right)^{3}
$$

and the result will be a numerical multiple of the contravariant $d x^{3}+\ldots-a y^{3}$. If, in the above instance, we denote the square of the primitive and its skew covariant according to their degree and order by $2 \cdot 6,3 \cdot 3$ respectively, we may explain their mutual action stenographically by saying that $\dot{2} 6$ and $3 \dot{3}$ have acted reciprocally on each other, the dot signifying that the quantities typified by the number so marked have been replaced by their symbolic inverses; we cannot well represent this mutual action by writing $\dot{2} \cdot 6 * 3 \cdot \dot{3}$ or $\dot{2} 6$

$3 \cdot \dot{3} * \dot{2} \cdot 6$, but may employ for the purpose $*$. So from the square of a quintic $3 \cdot \dot{3}$

$\dot{2} \cdot 10$

$2 \cdot 10$ and its linear covariant $5 \cdot 1$ we may derive by reciprocal action $*$, or $5 \cdot \mathrm{i}$

the contravariant $3.9:$ or, again, we may take any even number of covariants and cause them to operate in various manners, the variables on the variables and the coefficients on the coefficients, so as to form a closed circuit, as, for example, with four, we may make the coefficients of the first operate on those of the second, the variables of the second on those of the third, the coefficients of the third on those of the fourth, and the variables of the fourth on those of the first. Thus we have passed from reciprocal to the more general notion of simultaneous or circulatory action between any even number of covariants. And it is not unlikely that further applications may be made of this fertile conception: when dealing with a principle (an intellectual force) as distinguished from a theorem (a mere law), we never can feel sure that its uses are exhausted, or its plastic power spent. 